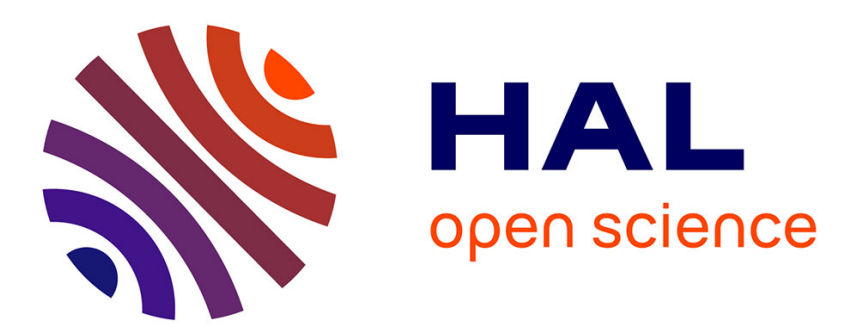

\title{
Numerical simulation of retention and release of colloids in porous media at the pore scale
}

\author{
Nisrine Sefrioui, Azita Ahmadi, Aziz Omari, Henri Bertin
}

\section{To cite this version:}

Nisrine Sefrioui, Azita Ahmadi, Aziz Omari, Henri Bertin. Numerical simulation of retention and release of colloids in porous media at the pore scale. Colloids and Surfaces A: Physicochemical and Engineering Aspects, 2013, 427, pp.33-40. 10.1016/j.colsurfa.2013.03.005 . hal-01081224

\section{HAL Id: hal-01081224 \\ https://hal.science/hal-01081224}

Submitted on 7 Nov 2014

HAL is a multi-disciplinary open access archive for the deposit and dissemination of scientific research documents, whether they are published or not. The documents may come from teaching and research institutions in France or abroad, or from public or private research centers.
L'archive ouverte pluridisciplinaire $\mathbf{H A L}$, est destinée au dépôt et à la diffusion de documents scientifiques de niveau recherche, publiés ou non, émanant des établissements d'enseignement et de recherche français ou étrangers, des laboratoires publics ou privés. 


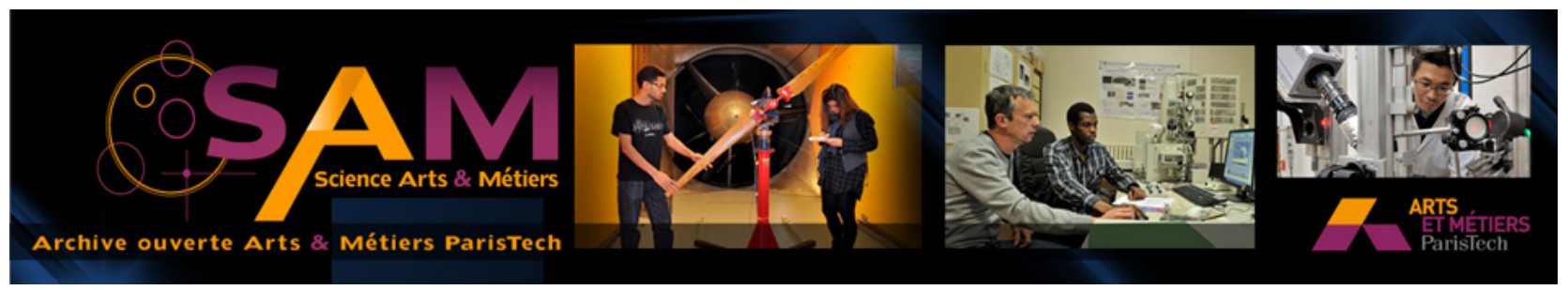

\section{Science Arts \& Métiers (SAM)}

is an open access repository that collects the work of Arts et Métiers ParisTech researchers and makes it freely available over the web where possible.

This is an author-deposited version published in: http://sam.ensam.eu

Handle ID: .http://hdl.handle.net/10985/8835

\section{To cite this version :}

Nisrine SEFRIOUI, Azita AHMADI, Aziz OMARI, Henri BERTIN - Numerical simulation of retention and release of colloids in porous media at the pore scale - Colloids and Surfaces A: Physicochemical and Engineering Aspects - Vol. 427, p.33-40 - 2013 


\title{
Numerical simulation of retention and release of colloids in porous media at the pore scale
}

\author{
Nisrine Sefrioui, Azita Ahmadi, Aziz Omari, Henri Bertin* \\ I2M-TREFLE-UMR CNRS 5295, University of Bordeaux, Arts et Metiers ParisTech, Esplanade des Arts et Metiers, 33405, Talence, France
}

\section{H I G H L I G H T S}

- Direct Numerical Simulation has been performed.

- Different roughness types have been investigated.

- Physicochemical interactions have been included in the model.

- Residence time depend on roughness, hydrodynamics and physicochemical interactions.

\section{G R A P H I C A L A B S T R A C T}

Flow structure and particle trajectory for a valley geometry roughness (dashed line corresponds to ideal trajectory).

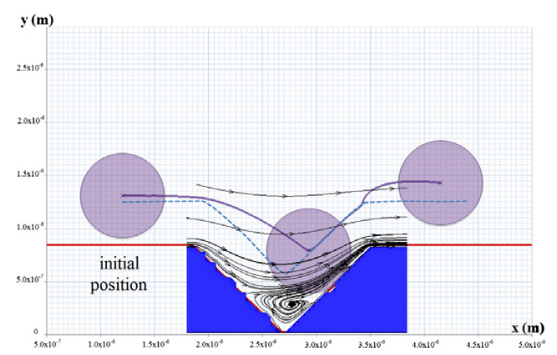

\section{A B S T R A C T}

Transport of a solid colloidal particle was simulated at the pore scale in presence of surface roughness and particle/pore physicochemical interaction by adopting a "one fluid" approach. A code developed in our laboratory was used to solve equations of motion, while implementing additional modules in order to take into account lubrication and physicochemical forces. Particles were recognized through a phase indicator function and the particle/fluid interface position at each instant was obtained by solving a transport equation. Roughnesses of different shapes were considered and the magnitude of the particle/pore physicochemical interaction was monitored through the change of the ionic strength of the suspending fluid. We first show that if pore surface is smooth no retention of the transported particle occurs whether the particle/pore surface is attractive or repulsive. However for shape roughnesses of "peak" or "valley", particles may be retained inside pores or not depending on the considered ionic strength. In absence of particle retention, the residence time (the time needed for a particle to travel a characteristic pore distance) is finite and was found to be an increasing function of ionic strength for every considered roughness at fixed hydrodynamic conditions.

\section{Introduction}

Flow of reactive solutions or suspensions in saturated porous media is of great interest in many environmentally relevant applications such as contaminant dissemination, filtration and remediation processes. In such processes, the determination of the

\footnotetext{
* Corresponding author. Tel.: +33 5568454 06; fax: +33 556845436.

E-mail address: h.bertin@i2m.u-bordeaux1.fr (H. Bertin).
}

concentration of species as a function of time and space is of major concern [1]. It is admitted that besides porous media structure, transport and chemical aspects are the most important phenomena that should be considered in interpreting field and laboratory data or in analyzing modeling results.

Considering chemical aspects, when reactive species are charged colloid particles of finite size, the DLVO (Derjaguin-Landau-Verwey-Overbeek) theory is often put forward to describe particle/pore surface interaction. If such an interaction is purely attractive the adsorption conditions are called 
favorable with no energy barrier while unfavorable conditions are reserved to non-monotonic interaction pr ofiles which generally present an energy barrier, a deep primary minimum and a shallow secondary minimum. Under physicochemical conditions such that unfavorable conditions are expected to prevail, the calculated energy barrier is usually high enough (several hundreds of $\mathrm{kT}$ ) so that colloid adsorption should be precluded. However, this behavior was reported to be in contrast with experimental evidences showing a significant adsorption. To explain such apparent discrepancy several possible sources were examined. Among them, the predominant role of the secondary minimum in the adsorption process was emphasized [2-4] and it was shown that on the basis of this, the influence of physicochemical parameters as ionic strength and $\mathrm{pH}$ of the background solution were well predicted [2]. Nevertheless despite qualitative theory/experiment agreement, quantitative discrepancies were reported to persist. It was therefore argued that as particle and pore wall surfaces are not chemically and/or topographically homogenous, a more precise calculation of interaction potentials on small scale is needed for quantitative comparison. Chemical heterogeneity is introduced through the existence of nano-sized chemical patches whose composition may locally induce irreversible adsorption of particles in the primary minimum [5,6]. In that respect, several models containing two or more classes of adsorption sites were proposed in the literature [7-11]. The local pore structure and surface topography heterogeneity considered as asperities were shown to induce a shift of the actual interaction potential. Indeed, repulsive interaction between a colloidal particle and a solid surface is lower on a rough surface compared to a smooth surface [12-14]. The extent of this interaction depends mainly on the asperities characteristic height $H$, their form and the inter-asperities distance relative to particle size. Moreover, these topographic heterogeneities play an important role in both particle retention and its counterpart particle release phenomena through flow pattern modification. When a porous medium is modeled as a collection of spherical grains, non-deformable colloid particles are considered to firstly adsorb onto grain surface and may subsequently roll on it under hydrodynamic drag force and/or diffusion and then are retained by accumulation in stagnation regions at grain-grain contact zone or in the rear of the grain [15-18]. Similarly colloid particles may be re-entrained by escaping from these retention regions going back to the bulk flowing suspension (re-suspension mechanism). This was introduced in the convection-dispersion equation [19] in order to simulate experimental breakthrough curves but too many rate dependent constants had to be adjusted.

Basically, detachment of an adsorbed particle results from a balance between external forces exerted on it. These are adhesion (physicochemical interactions), drag and lift forces. So, depending on whether the particle is hard (slightly deformable) or soft (highly deformable), an adapted continuum mechanics model is usually used to predict the physicochemistry/hydrodynamic relationship that governs particle detachment. For smooth surfaces, major studies predict that detachment should occur mainly by rolling [20-23]. Bergendahl and Grasso [22] have shown that incipience of particle rolling is well correlated to a dimensionless parameter $N_{\mathrm{TFT}}$; the ratio of the depth of the primary minimum to the exerted shear force torque. The roughness of grain surface was earlier considered by several authors [24-27]. Burdick et al. [26] studied the behavior of colloids of given sizes in the vicinity of an obstacle. Examination of balance of forces and torques that are exerted on a retained deformable colloid showed that its re-entrainment follows a lift process rather than a rolling which is expected to predominate in the removal of particles adsorbed on smooth surfaces. By the same way, Neyland [28] examined the influence of both the height (and the depth) of asperities and their separation distance on critical flow rate for particle re-entrainment. However, the problem considered was only in 2 dimensions and the discussion of results was only qualitative.

From what is briefly exposed here, it may be seen that representative simulations of retention and removal of colloidal particles of given size in presence of a rough surface is still lacking. So, the goal of this paper is to propose a numerical modeling of retention and re-entrainment of micron sized colloids in presence of roughness on a grain surface. In this work, a direct numerical simulation method was adopted including both hydrodynamic and physicochemical interactions in the vicinity of asperities of various shapes and characteristic sizes. In the next section the adopted method is described before presentation in section 3 of primary results showing successively the influence of physicochemical conditions through the ionic strength variations and hydrodynamic conditions all in presence of a rough or a smooth surface.

\section{Numerical model}

\subsection{Configuration}

The geometrical configuration and physical parameters chosen for this study are based on recent experiments [29,30]. In those works, deposition and release of negatively charged colloidal latex particles of a radius, $a_{\mathrm{p}}$, of $400 \mathrm{~nm}$ in an artificial sintered silicate porous medium were investigated.

Hydrodynamic parameters are also based on laboratory experiments $[29,30]$. The inlet velocity used in the simulations is equal to $4 \times 10^{-5} \mathrm{~ms}^{-1}$ leading to the following values of the Péclet number and the Particulate Reynolds number respectively:

$P_{\mathrm{e}}=\frac{V_{\mathrm{p}} a_{\mathrm{p}}}{D_{\mathrm{b}}}=9.8$

$R e_{\mathrm{p}}=\frac{\rho_{\mathrm{p}} a_{\mathrm{p}} \bar{u}}{\mu}=1.66 \times 10^{-5}$

where $V_{\mathrm{p}}$ is the particle velocity corresponding to the fluid velocity evaluated at a distance of $3 a_{\mathrm{p}}$ from the grain surface, $D_{\mathrm{b}}$ is the bulk particle diffusion coefficient. $\rho_{\mathrm{p}}, \bar{u}$ and $\mu$ are respectively the particle density, the interstitial velocity and the dynamic viscosity of the fluid.

For given $P_{\mathrm{e}}$ and $R e_{p}$ and for each roughness, three different values of salinity $(0.5,1.2$ and 2 or $3 \mathrm{~g} / \mathrm{L}$ of $\mathrm{NaCl})$ are considered leading to three levels of ionic strength, I, designated by weak ( $\mathrm{I}=3 \mathrm{mM})$, medium $(\mathrm{I}=7.8 \mathrm{mM})$ and strong $(\mathrm{I}=12$ or $18 \mathrm{mM})$. These values of I correspond to DLVO forces of significantly different intensities (Fig. 1).

The domain chosen for this study is a rectangular prism (5 $\mu \mathrm{m} \times 4 \mu \mathrm{m} \times 3.8 \mu \mathrm{m})$, in contact with the grain surface (Fig. 2 ).

The topographic heterogeneities considered here are surface asperities on the grain surface. The roughness geometries are simple and have acute angles, which for a class of porous media such as sands can be closer to reality than hemispherical plots or asperities that are mostly used in the literature [12,25,31-33]. Therefore, besides the smooth surface, right triangular prisms of the form of peaks and valleys with two different sizes are considered. (Fig. 3) The heights, $H$, correspond to one or two times the particle radius, $a_{\mathrm{p}}$ and therefore vary from $-2 a_{\mathrm{p}}$ to $2 a_{\mathrm{p}}$. Despite the $2 \mathrm{D}$ nature of the asperities, particles are spherical and all simulations are performed in 3 dimensions. The colloid is placed on the symmetry plane of the domain in the thickness and the results will be presented in this plane.

The particle transport is solved by Direct Numerical Simulation (DNS) with fixed Cartesian grids. The size of the grid blocks are chosen so that the particle diameter contains 16 blocks. Previous works 


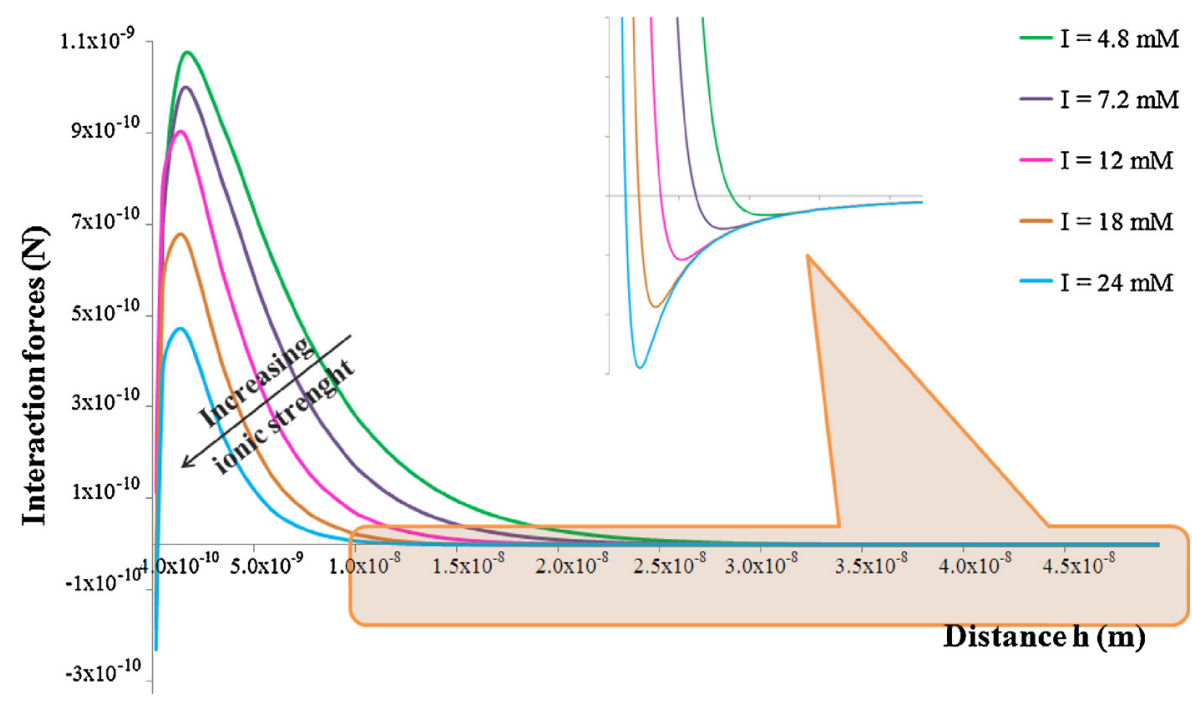

Fig. 1. DLVO Interaction forces for different ionic strength values as a function of surface-to-surface separation distance, $h$.

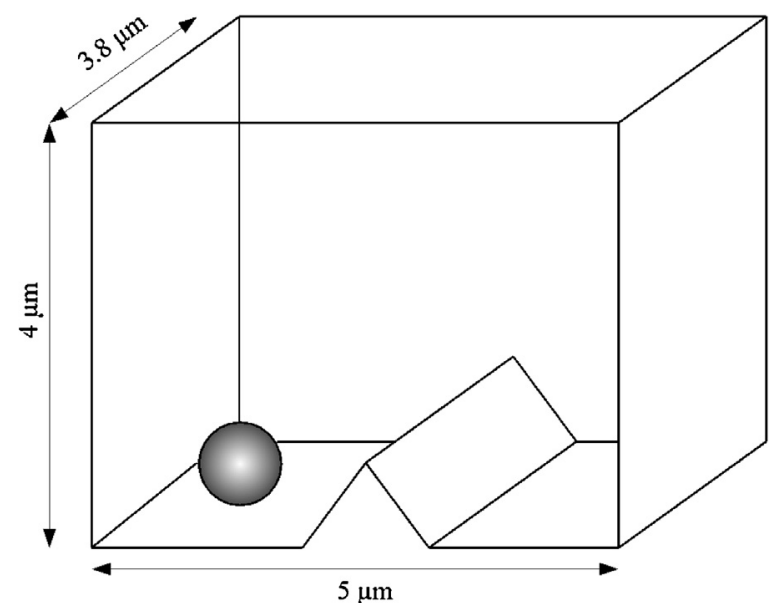

Fig. 2. Schematic view of the simulation domain (case of peak geometry). have shown that, with the numerical choices made here which will be presented in the next section, a number of grid blocks between 8 and 16 ensure numerical results in agreement with the physics of particle transport for a large range of Reynolds numbers [34,35].

\subsection{Model}

\subsubsection{Governing equations}

Simulations are performed using the numerical code Thetis ${ }^{\circledR}$, developed in our lab, in which additional modules have been added in order to take into account particle/particle and particle/grain surface physicochemical interactions. A generalized one-fluid model has been used for the transport of particles [35]. The entire domain is considered as fluid and the solid is a particular fluid with special properties, the two phases being distinguished through a phase indicator function, $F_{\mathrm{c}}$. The evolution of the particle is described by an advection equation on $F_{\mathrm{c}}$. The flow of the incompressible Newtonian fluid is governed by the Navier-Stokes and the mass balance equations. The final set of partial differential equations is given by the one-fluid model given below:

$$
\nabla \cdot \mathbf{u}=0
$$
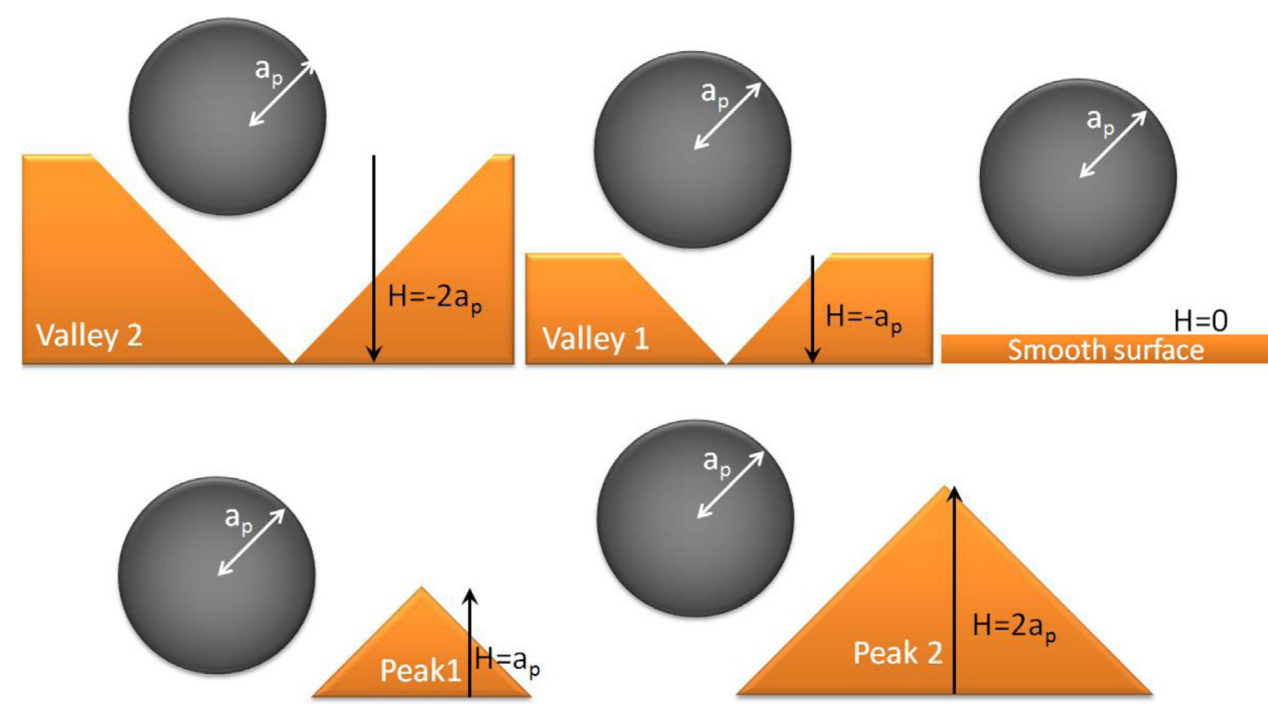

Fig. 3. Roughness geometries with respect to particle dimension $H \in\left[\left[-2 a_{\mathrm{p}} ; 2 a_{\mathrm{p}}\right]\right]$. 


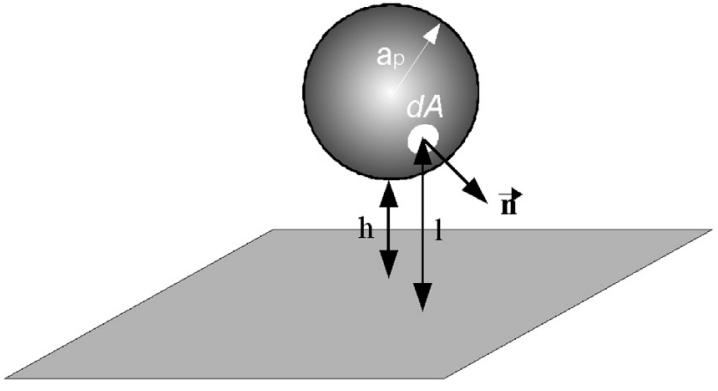

Fig. 4. Surface Element Integration between a spherical particle and a rough surface.

$\rho\left(\frac{\partial \mathbf{u}}{\partial t}+(\mathbf{u} \cdot \nabla) \mathbf{u}\right)=-\nabla p+\nabla \cdot\left(\mu\left(\nabla \mathbf{u}+\nabla^{t} \mathbf{u}\right)\right)+\mathbf{F}_{\mathbf{s}}$

$\frac{\partial F_{\mathrm{c}}}{\partial t}+\mathbf{u} \cdot \nabla F_{\mathrm{c}}+0$

where $\mathbf{u}, \rho, p$ and $\mathbf{F}_{\mathbf{s}}$ are respectively the velocity, the fluid density, the pressure, and a source term corresponding to volumetric volume forces. This force can include gravity, lubrication and DLVO. In this work, gravity is not taken into account and only particle-grain and particle-particle DLVO forces are added in Eq. (4). When the particle-grain or particle-particle distance is less than a grid size, hydrodynamic forces cannot be computed correctly. In that case, a lubrication force is also added in the term $\mathbf{F}_{\mathbf{s}}[34,35]$.

\subsubsection{DLVO forces}

Physicochemical interactions between particles or a particle and a grain surface include van der Waals (vdW) and electrical double layer (DL) forces, the sum of which is called the DLVO force (DLVO). For the case of a spherical particle near an homogeneous infinite flat plate (smooth grain surface in our case), the approximate analytical expressions of these forces denoted respectively $F_{\mathrm{vdW}}^{\mathrm{SP}}, F_{\mathrm{DL}}^{\mathrm{SP}}$ and $F_{\mathrm{DLVO}}^{\mathrm{SP}}$ are given by Prieve and Ruckenstein [36]:

$F_{\mathrm{vdW}}^{\mathrm{SP}}=-\frac{2 A_{\mathrm{H}} a_{\mathrm{P}}^{3}}{3 h^{2}\left(h+a_{\mathrm{p}}\right)^{2}}$

$F_{\mathrm{DL}}^{\mathrm{SP}}=\frac{2 \kappa \pi \varepsilon_{0} \varepsilon_{\mathrm{r}} a_{\mathrm{p}}}{1-e^{-2 \kappa h}}\left(2 \zeta_{\mathrm{P}} \zeta_{\mathrm{S}} e^{-\kappa h}-\left(\zeta_{\mathrm{P}}^{2}+\zeta_{\mathrm{S}}^{2}\right) e^{-2 \kappa h}\right)$

$F_{\mathrm{DLVO}}^{\mathrm{SP}}=F_{\mathrm{vdW}}^{\mathrm{SP}}+F_{\mathrm{DL}}^{\mathrm{SP}}$

where $A_{\mathrm{H}}$ is the particle/water/solid Hamaker constant; $h$ is the minimum separation distance between the particle and the flat plate; $\kappa$ is the inverse Debye screening length; $\varepsilon_{0}$ is the dielectric permittivity of vacuum; $\varepsilon_{\mathrm{r}}$ is the relative dielectric constant of water and $\zeta_{\mathrm{P}}$ and $\zeta_{\mathrm{S}}$ are the surface zeta potentials of the particle and the grain respectively. Eq. (7) is an approximation [37] that is assumed valid when $\kappa a_{\mathrm{p}} \gg 1$ and which is verified in the cases studied in this work. When the grain surface includes some kind of heterogeneity (chemical or topographic), the analytical expressions introduced above do not hold. It becomes important to take into account the surface roughness and curvature in the analytical expressions. In that case, many authors [12,37-39] replace the DLVO analytical expressions by using different approximations: Derjaguin Approximation Technique(DAT), Grid Surface Integration (GSI) and Surface Element Integration (SEI). These approximation techniques aim to obtain the total interaction energy between a particle and a given surface by integrating over the whole surface of the particle the interaction energy per unit area between a surface element of the particle and an element of the grain surface using the approximation obtained for two infinite flat plates ( $\left.E^{\text {PP }}\right)$ (Fig. 4).

In this work, the computation of DLVO forces between a spherical particle and a rough grain surface is performed by the SEI

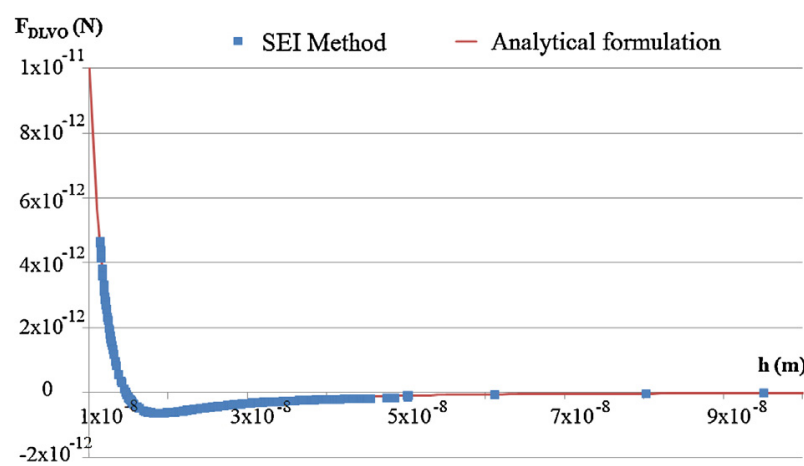

Fig. 5. DLVO force profiles between a colloidal particle ( $400 \mathrm{~nm}$ radius) and a smooth surface with varying separation distance.

method $[37,39]$. This method leads to the expression of the DLVO force $\left(F_{\mathrm{DLVO}}^{\mathrm{SEI}}\right)$ based on the integration of the sum of the derivatives of plate-plate van der Waals $\left(E_{\mathrm{vdW}}^{\mathrm{PP}}\right)$ and double layer $\left(E_{\mathrm{DL}}^{\mathrm{PP}}\right)$ energies per unit area, given below for a varying separation distance 1 [39]:

$E_{\mathrm{vdW}}^{\mathrm{PP}}(l)=-\frac{A_{\mathrm{H}}}{12 \pi l^{2}}$

$E_{\mathrm{DL}}^{\mathrm{PP}}(l)=\frac{\varepsilon_{0} \varepsilon_{\mathrm{r}} \kappa}{2}\left(\zeta_{\mathrm{P}}^{2}+\zeta_{\mathrm{S}}^{2}\right)\left[1-\operatorname{coth}(\kappa l)+\frac{2 \zeta_{\mathrm{P}} \zeta_{\mathrm{S}}}{\left(\zeta_{\mathrm{P}}^{2}+\zeta_{\mathrm{S}}^{2}\right)} \operatorname{csch}(\kappa l)\right]$

$F_{\mathrm{DLVO}}^{\mathrm{SEI}}=\iint_{\text {sphere }}\left(-\frac{\partial E_{\mathrm{vdW}}^{\mathrm{PP}}(l)}{\partial l}-\frac{\partial E_{\mathrm{DL}}^{\mathrm{PP}}(l)}{\partial l}\right) \mathbf{n}^{\prime} \cdot \mathbf{e}_{2} d A$

where $\mathbf{n}^{\prime}$ is the normal unit vector outgoing from a surface element, $\mathbf{e}_{2}$ is the normal unit vector in the vertical direction and $d A$ is the differential area of a surface element.

The SEI method has been implemented and the results obtained for the particular case of a hard sphere/flat plate configuration were verified to be in good agreement with the analytical result obtained using Eqs. (6)-(8). Fig. 5 shows that for an ionic strength of $24 \mathrm{mM}$, the obtained results are in good agreement using 4500 elements even with separation distances less than the mesh size of a grid block. One must note that the goal of developing the SEI method is to compute DLVO forces for rough surfaces where the analytical formula does not hold anymore (Fig. 6).

\subsubsection{Lubrication forces}

When the separation distance between a particle and a plane or two particles is less than a limit distance, $h_{0}$, comparable to the size of a grid block, the Navier-Stokes equations cannot be solved correctly. At such a distance, the hydrodynamic forces acting on the sphere are underestimated. In this case, a lubrication force $\mathbf{F}_{\text {lub }}$ has to be added to the term $\mathbf{F}_{\mathbf{s}}$ in Eq. (4) [40-42]. The normal component of this force which is involved in particle contact with the wall is written as:

$\mathbf{F}_{l u b} \cdot \mathbf{n}=-6 \pi \mu a_{\mathrm{p}}\left[\lambda_{s}\left(\varepsilon_{h}\right)-\lambda_{s}\left(\varepsilon_{h_{0}}\right)\right] \mathbf{v}_{\mathrm{p}} . \mathbf{n}$ for $h \leq h_{0}$

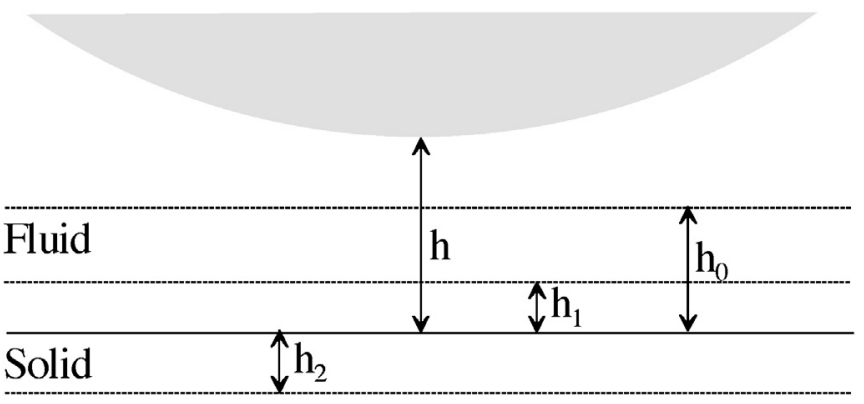

Fig. 6. Multi layer model used for lubrication interaction. 
where $\varepsilon_{h}=h / a_{\mathrm{p}}$ is a dimensionless surface-to-surface distance between neighboring particles or between a particle and a grain surface, $\varepsilon_{h_{0}}=h_{0} / a_{\mathrm{p}}$, is the dimensionless critical distance for activation or deactivation of lubrication, $\mathbf{v}_{\mathrm{p}}$ is the particle velocity, $\mathbf{n}$ is the normal unit vector directed from the particle towards the plane or another particle and $\lambda_{s}$ is the Stokes correction (amplification) factor. Its estimation is very important to capture the right physics in the close vicinity of the grain surface $\left(\varepsilon_{h} \leq \varepsilon_{h_{0}}\right)$ and its formulation for a particle-wall interaction is usually written as:

$\lambda_{s}\left(\varepsilon_{h}\right)=\frac{1}{\varepsilon_{h}}-\left(\frac{1}{5}+\frac{1}{21} \varepsilon_{h}\right) \log \left(\varepsilon_{h}\right)+0.9713+0\left(\varepsilon_{h}\right)$

The lubrication interaction is activated when the dimensionless separation distance $\varepsilon_{h}$ becomes less than the critical value, $\varepsilon_{h_{0}}$, and is kept constant for $0<\varepsilon_{h}<\varepsilon_{h_{1}}$. When the particle overlaps with the wall $\left(\varepsilon_{h_{2}}<\varepsilon_{h}<\varepsilon_{h_{1}}\right)$ a soft sphere model for solid contact is introduced in order to reject the particle towards the fluid phase. The parameters $\varepsilon_{h_{0}}, \varepsilon_{h_{1}}$ and $\varepsilon_{h_{2}}$ must be adjusted to obtain physical solutions. This procedure is described in details elsewhere [34] and was proven to predict properly the restitution coefficient for a particle colliding normally a solid wall. Therefore, by doing so we account successively for long range hydrodynamic interactions by solving the Navier-Stokes equations, the short range lubrication effect and the solid-solid collision.

\subsection{Simulation method}

The numerical model used by Thetis ${ }^{\circledR}$ is based on an implicit tensorial penalty method [43]. In this method, a special decomposition of the stress tensor, $\sigma$, is proposed:

$\sigma=-p \mathbf{I}+\iota \mathbf{\Lambda}+\chi \boldsymbol{\gamma}+\omega \boldsymbol{\Theta}$

where $\iota, \chi$ and $\omega$ are respectively the elongation, the shearing and the rotation viscosities. The tensors $\Lambda, \gamma$ and $\Theta$ are respectively given by:

$$
\begin{gathered}
\boldsymbol{\Lambda}=\left[\begin{array}{ccc}
\frac{\partial u_{x}}{\partial x} & 0 & 0 \\
0 & \frac{\partial u_{y}}{\partial y} & 0 \\
0 & 0 & \frac{\partial u_{x}}{\partial z}
\end{array}\right] ; \quad \boldsymbol{\gamma}=\left[\begin{array}{lll}
0 & \frac{\partial u_{x}}{\partial y} & \frac{\partial u_{x}}{\partial z} \\
\frac{\partial u_{y}}{\partial x} & 0 & \frac{\partial u_{y}}{\partial z} \\
\frac{\partial u_{z}}{\partial x} & \frac{\partial u_{z}}{\partial y} & 0
\end{array}\right] ; \\
\boldsymbol{\Theta}=\left[\begin{array}{lll}
0 & \frac{\partial u_{x}}{\partial y}-\frac{\partial u_{x}}{\partial x} & \frac{\partial u_{x}}{\partial z}-\frac{\partial u_{x}}{\partial x} \\
\frac{\partial u_{y}}{\partial x}-\frac{\partial u_{y}}{\partial y} & 0 & \frac{\partial u_{y}}{\partial z}-\frac{\partial u_{y}}{\partial y} \\
\frac{\partial u_{z}}{\partial x}-\frac{\partial u_{z}}{\partial z} & \frac{\partial u_{z}}{\partial y}-\frac{\partial u_{z}}{\partial z} & 0
\end{array}\right]
\end{gathered}
$$

where $u_{x}, u_{y}$ and $u_{z}$ are respectively the components of the local fluid velocity vector in the $x, y$ and $z$ directions.

The choice of the different viscosities ensures physical characteristics of the fluid and the solid phases. In this manner, one can impose incompressibility of the fluid and undeformability of the solid. The reformulated Navier-Stokes equations are solved using a finite volume method on staggered grids, where a second order centered approximation scheme is used. The algorithm used is based on the augmented Lagrangian method which allows coupling the velocity and the pressure while keeping an implicit method.

The particle is reconstructed using an interface tracking method. Grid blocks are fixed throughout the simulations and solid or fluid phases are identified by their characteristics using the phase indicator function, $F_{\mathrm{c}}$. The scheme used for the resolution of the advection of the indicator function is an Eulerian Langrangian (VOF) Volume of Fluid method. An explicit scheme has been used; its stability is ensured by a CFL condition which limits the time steps. Indeed, the particle is not allowed to travel through a grid block during a time step. The existence of DLVO forces at short separation distances, with high intensities can lead to large particle velocities and therefore very small time steps. This has lead to very large computation times going from a few days to a few weeks on a cluster of 256 cores.

The boundary conditions imposed are: constant flow rate at the inlet and a Neumann condition on the velocity at the outlet. The latter corresponds to imposing $\nabla u_{i} \cdot \mathbf{n}=0 \quad(i=x, y, z)$ with $\mathbf{n}$ unit vector normal to the outlet face. No-slip boundary conditions are imposed on the lateral and bottom surfaces and a slip velocity was imposed at the top surface of the domain in order to have a less confined region despite the small domain size. One should note that in the model Thetis ${ }^{\circledR}$, there is no need for a boundary condition in terms of pressure in order to capture the physics of the flow. The pressure is calculated with respect to a reference pressure and is therefore a relative value.

During a simulation, pressure, velocity and $F_{\mathrm{c}}$ are stored. This allows to study the structure of the fluid flow and to follow the particle trajectory. This is done for different physicochemical conditions and grain roughness with a particular interest for cases where the simulation conditions lead to particle retention. Additional data, such as hydrodynamic and DLVO forces at each time step are also computed allowing a more detailed analysis of the particle behavior. In order to relate these simple simulations to macroscopic results, residence time estimation has been performed as a first approach.

\section{Results and discussion}

\subsection{Flow structure}

The flow structure for peak geometry is first presented in Fig. 7a where flow pattern and pressure field in the suspending fluid are superimposed. It may be seen that both velocity and pressure fields are disturbed by the solid obstacle representing the roughness and the moving hard spherical particle. Flow streamlines are globally as expected packing down in the immediate vicinity of the solid particle. The formation of a stagnation zone in the rear of the obstacle must also be noted. This means that during the transport of very small negligibly diffusing particles, their centre of mass would follow the flow streamlines and they would be transported far behind the solid obstacle. Such an exclusion zone is also expected to exist during adsorption process of new flowing colloidal particles in the vicinity of already adsorbed particles and is referred to as the hydrodynamic shadowing effect. This effect is obviously more pronounced as flow strength increases [44]. Concerning the pressure field, an overpressure is localized in the upstream of the hard sphere and a depressive wake in its downstream similar to the classic case where a hard sphere is imbedded in an imposed uniform flow. In Fig. 7b the velocity field readily computed in the particle itself is presented. The particle undergoes both translation and rotation due to the surrounding fluid. It is worth noting that under the same conditions, less flow perturbation is noticed by decreasing the peak height as expected (data not shown) but such a perturbation is strongly changed when the valley form is considered. The flow for this latter geometry will be illustrated and commented in the next section.

\subsection{Trajectory versus ionic strength for given Re}

In this section, particle trajectories are studied under fixed hydrodynamic conditions and by changing physicochemical interaction through variation of the ionic strength of the suspending 


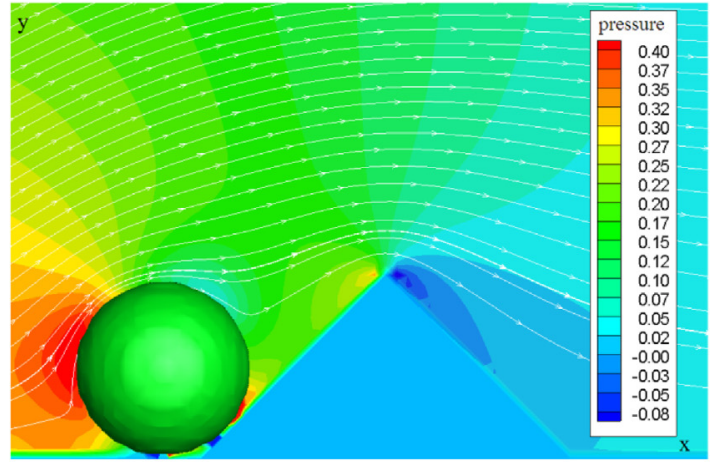

a

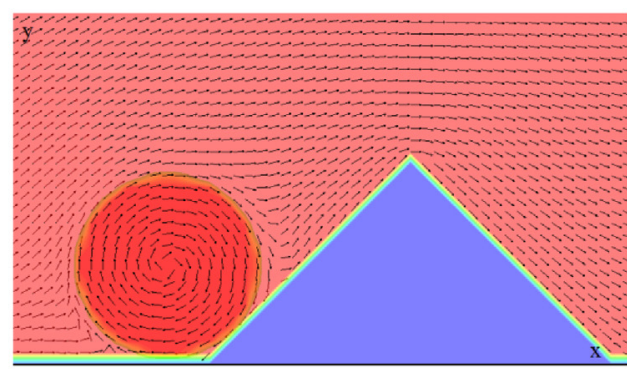

$\mathrm{b}$

Fig. 7. Pressure and streamlines (left) and velocity fields (right) close to a peak geometry.

fluid. Indeed, the influence of suspending fluid $\mathrm{pH}$ has to be considered as it determines the value of both Debye length and zeta potentials that consequently modify the interaction potential. However, in practice, $\mathrm{pH}$ value is imposed by the porous medium itself (buffering effect), rather than being considered as a variable parameter.

\subsection{Smooth flat grain surface}

First, the reference case of a smooth flat grain surface is considered. In that case, whatever the ionic strength and therefore the magnitude of the physicochemical interaction force, injected particles mainly follow a straight trajectory parallel to the grain surface. The only difference from one situation to another is the initial position of particle versus the interaction intensity. This leads to no particles retention and a finite travel time that increases as ionic strength increases. Indeed, for a low ionic strength, the particle-pore surface interaction potential presents a significant energy barrier prohibiting the particle to come in close contact with the solid surface and is pushed away from the surface. When I is increased, the energy barrier decreases enabling particles to remain in the neighborhood of the solid surface leading hence to a lower velocity.

\subsection{Peak shaped roughness}

With the same considerations and in presence of a peak-shaped roughness, we may reasonably expect at low ionic strength a moving particle to jump over the roughness and be transported far away from the obstacle. Again by increasing the ionic strength both the energy barrier height and its distance range are reduced and the particle is allowed to approach the solid surface more closely. This is clearly shown by inspecting the trajectory corresponding to medium ionic strength for which the particle is seen to come back in contact with solid surface after over passing the roughness and then continues to move while rotating (Fig. 8). If the ionic strength is further increased, it may be so that the particle-solid wall interaction is attractive at similar separation distances (see secondary minimum in Fig. 1). This is what is readily observed for the high I value where the particle, after a displacement period, is stopped at the foot of the peak and the particle retained does not move any more. Once again comparable results are obtained for the smaller peak in the sense that for moderate and low ionic strength values, the particle flows over the asperity and it is retained for the high I value

\subsection{Valley shaped roughness}

Similar in silico experiments have been carried out in case of the valley geometry at the same Reynolds number. Typical flow pattern is shown in Fig. 7 where a region of close streamlines is clearly seen in the bottom of the valley. This situation is expected to occur at grain-grain contacts in case of unconsolidated porous media and there is a large zone that may only be explored by diffusion by colloidal particles of very small size. As a consequence, the actual trajectory of particle differs significantly from that following the solid surface as it may be seen in Fig. 9. Such experiment was repeated as previously for different I values and once again no particle retention was observed for low and intermediate ionic strength. For the high ionic strength the particle is trapped in the valley due to attractive physicochemical interaction in an analogous way to what happens in presence of peak asperities. However if such retention was observed in peak cases for the two values of the height considered, here colloid retention was only observed for the deeper valley. Such difference arises from the fact that in the case of peaks, the particle interacts with solid wall via two points whereas only one interaction point exist in case of valleys under our hydrodynamic conditions.

From these experiments it may be concluded that under given hydrodynamic conditions, the roughness characteristics and physicochemical conditions play major roles in colloid retention in porous media. They also suggest that peak shaped roughnesses are more efficient in retention of flowing colloids in porous media rather than valleys. In any case, our simulations show clearly that a rough surface is quite necessary to provoke retention of non inert colloids in porous media even if that retention depends on grain surface topography.

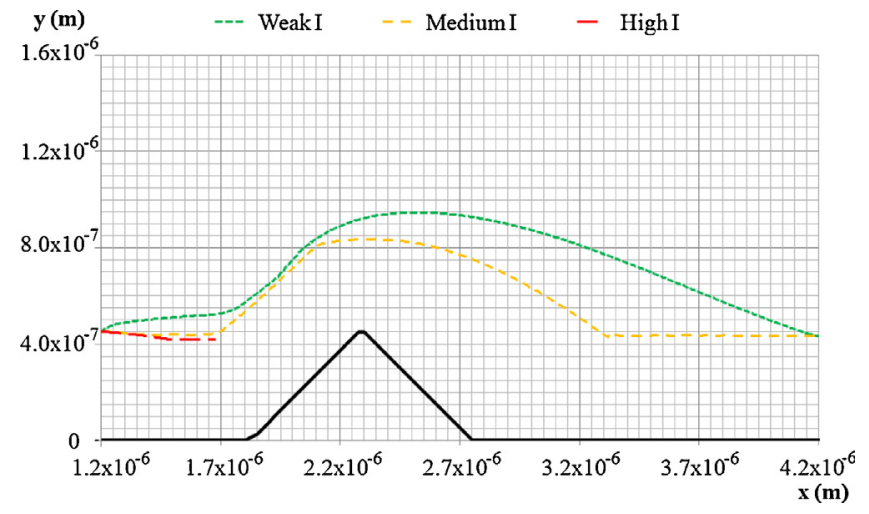

Fig. 8. Particle trajectories for the small peak $\left(H=a_{\mathrm{p}}\right)$ for different values of ionic strength. 


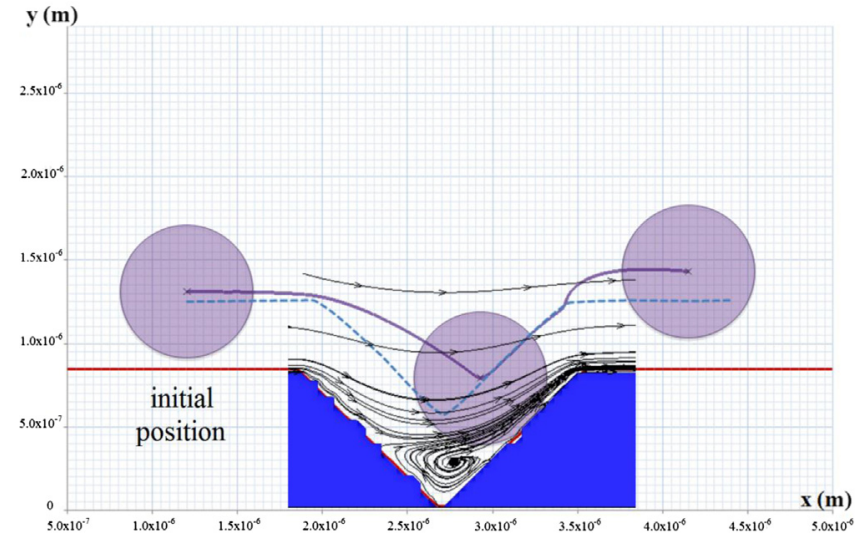

Fig. 9. Flow structure and particle trajectory for a valley geometry roughness (dashed line corresponds to ideal trajectory).

\subsection{Dimensionless analysis}

After presentation and general comments of predicted influence of ionic strength on flow structure and colloids retention in presence of roughnesses of different shapes and sizes, in this paragraph we compare the results obtained for all considered geometry cases. Up to now the imposed flow rate was often kept constant and consequently we still set aside quantitative study of the role of hydrodynamic interactions and their competition with physicochemical interactions. Nevertheless and anticipating forthcoming simulations, we are mainly interested in the dependence of the residence time, $\tau$, defined here as the time needed to travel through a fixed domain containing the roughness versus a dimensionless number that encompasses hydrodynamic and physicochemical interactions. For that purpose, let us first consider the Newton's second law of motion written in a dimensionless form as:

$R e_{\mathrm{p}} \frac{d \mathbf{v}_{\mathbf{p}}^{*}}{d t^{*}} \mathbf{F}_{\text {Hydro }}^{*}+N_{1} \mathbf{F}_{\mathbf{v d \mathbf { W }}}^{*}+N_{2} \mathbf{F}_{\mathbf{D L}}^{*}$

where

$N_{1}=\frac{\kappa A_{\mathrm{H}}}{\mu a_{\mathrm{p}} \bar{u}}$

$N_{2}=\frac{\varepsilon_{0} \varepsilon_{\mathrm{r}} \zeta_{\mathrm{P}} \zeta_{\mathrm{S}}}{\mu a_{\mathrm{p}} \bar{u}}$

Both of these dimensionless numbers depend on particle radius and, in an identical manner on the mean flow velocity. However, when they are plotted against I using experimental data that correspond to the experimental system considered as a reference [29], $N_{2}$ was found to be more rapidly varying than $N_{1}$. Moreover, while $N_{1}$ includes electrostatic interactions through $\kappa$ and van der Walls interactions through the Hamaker constant $A_{\mathrm{H}}$, only electrostatic parameters such as $\xi_{\mathrm{p}}$ and $\xi_{\text {s }}$ are present in $N_{2}$ which depends hence exclusively on the background solution chemistry and flow intensity. In Fig. 10 the variation of the dimensionless reciprocal residence time $\tau^{-1^{*}}$ as a function of $N_{2}$ is therefore plotted for every topography and ionic strength, the Reynolds number being constant. Note that reference time corresponds to the one obtained for a flat surface and zero ionic strength.

It is first observed that whatever the shape and magnitude of considered roughness, the residence time globally decreases as $\mathrm{N}_{2}$ increases (or ionic strength decreases) and this was qualitatively explained before. Secondly, it is shown that for some roughnesses, $\tau$ is infinite at low $N_{2}$ (high ionic strength) meaning a retention of injected colloids (see the insert in Fig. 10). However for smooth grain surface, the residence time is always finite and no retention is thus predicted. In such a situation the non deformable colloidal

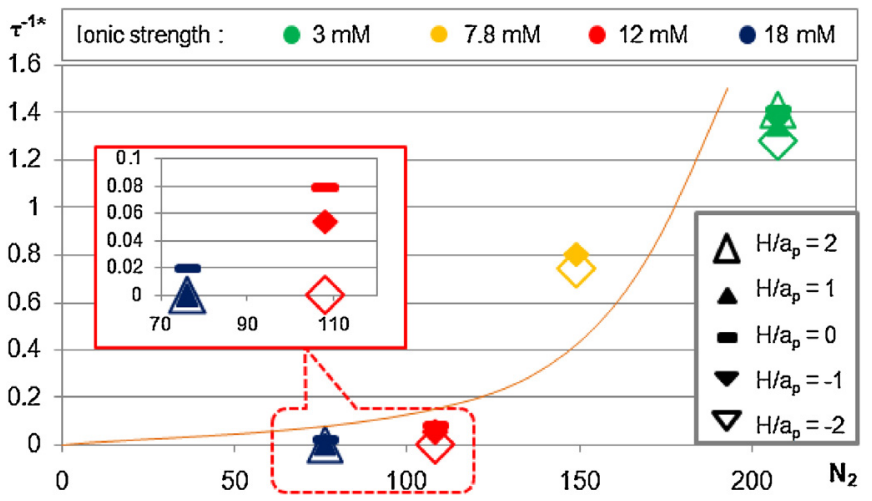

Fig. 10. Dimensionless reciprocal residence time as a function of dimensionless number $\mathrm{N}_{2}$ for different values of ionic strength and roughness geometries.

sphere does interact with the solid grain only via a "contact point" and the attractive force is oriented toward the centre of the sphere (and perpendicular to mean flow streamlines). This implies that the colloid can freely rotate and can then always escape from the domain within a finite lapse of time. In other words, for a colloid to be adsorbed on a smooth surface, the particle and/or the grain should be deformed when they come in close contact creating a contact area. In that case, it is accepted that a sufficient hydrodynamic torque is needed to remove the colloid and the magnitude of such a torque is dependent on the mechanical characteristics of the materials involved beside the prevailing hydrodynamic and physicochemical conditions [6,21-26].

\section{Conclusion}

In this work, additional modules were implemented in a fluid flow tool in order to simulate colloid transport at the pore scale in presence of rough pore surface while taking into account physicochemical interactions. For non deformable particles it was shown that the existence of surface roughness is a necessary but not sufficient condition for particles retention. For a fixed Reynolds number and under conditions for which no retention occurs, the residence time was found to increase with increasing ionic strength as the particle/pore surface becomes less repulsive. Moreover and from a dimensional analysis we expect the residence time to scale with a dimensionless number that encompasses both hydrodynamic and ionic strength effects. Additional simulations for constant ionic strength of the suspending fluid and various values of Reynolds number must be performed in order to fully validate the analysis.

As a long-term perspective and to be representative of real experiments, simulations should also be carried out using a given distribution of true 3D roughness since the characteristic length of the topography (the wave length) is expected to play a major role both in particle retention and release.

\section{References}

[1] M.A. Mojid, H. Vereecken, On the physical meaning of retardation factor and velocity of a nonlinearly sorbing solute, J. Hydrol. 302 (1-4) (2005) 127-136.

[2] M.W. Hahn, C.R. O'Melia, Deposition and re-entrainment of brownian particles in porous media under unfavourable chemical conditions: some concepts and applications, Environ. Sci. Technol. 38 (1) (2004) 210-220.

[3] J.A. Redman, S.L. Walker, M. Elimelech, Bacterial adhesion and transport in porous media: role of the secondary energy minimum, Environ. Sci. Technol. 38 (6) (2004) 1777-1785.

[4] M.W.Hahn, D. Abadzic, C.R. O'Melia, Aquasols: on the role of secondary minima Environ. Sci. Technol. 38 (22) (2004) 5915-5924.

[5] M. Tong, T.A. Camesano, W.P. Johnson, Spatial variation in deposition rate coefficients of an adhesion-deficient bacterial strain in quartz sand, Environ. Sci. Technol. 39 (10) (2005) 3679-3687. 
[6] M. Tong, X. Li, C.N. Brow, W.P. Johnson, Detachment-influenced transport of an adhesion-deficient bacterial strain within water-reactive porous media, Environ. Sci. Technol. 39 (10) (2005) 2500-2508.

[7] X. Li, T.D. Scheibe, W.P. Johnson, Apparent decreases in colloid deposition rate coefficients with distance of transport under unfavourable deposition conditions: a general phenomenon, Environ. Sci. Technol. 38 (21) (2004) 5616-5625.

[8] X. Li, W.P. Johnson, Nonmonotonic variations in deposition rate coefficients of microspheres in porous media under unfavourable deposition conditions, Environ. Sci. Technol. 39 (6) (2005) 1658-1665.

[9] N. Tufenkji, J.A. Redman, M. Elimelech, Interpreting deposition patterns of microbial particles in laboratory-scale column experiments, Environ. Sci. Technol. 37 (3) (2003) 616-623.

[10] N. Tufenkji, M. Elimelech, Deviation from the classical colloid filtration theory in the presence of repulsive DLVO interactions, Langmuir 20 (25) (2004) 10818-10828.

[11] N. Tufenkji, M. Elimelech, Spatial distributions of Cryptosporidium oocysts in porous media: evidence of dual mode deposition, Environ. Sci. Technol. 39(10) (2005) 3620-3629.

[12] E.M.V. Hoek, S. Bhattacharjee, M. Elimelech:, Effect of membrane surface roughness on colloid-membrane DLVO interactions, Langmuir 19 (11) (2003) 4836-4847.

[13] E.M.V. Hoek, G.K. Agarwal, Extended DLVO interactions between spherical particles and rough surfaces, J. Colloid Interface Sci. 298 (1) (2006) 50-58.

[14] C. Shen, V. Lazouskaya, H. Zhang, F. Wang, B. Li, Y. Jin, Y. Huang, Theoretical and experimental investigation of detachment of colloids from rough collector surfaces, Colloids Surf. 410 (2012) 98-110.

[15] S. Torkzaban, H.N. Kim, J. Simunek, S. Bradford, Hysteresis of colloid retention and release in saturated porous media during transients in solution chemistry, Environ. Sci. Technol. 44 (5) (2010) 1662-1669.

[16] X. Li, C.-L. Lin, J.D. Miller, W.P. Johnson, Pore-scale observation of microsphere deposition at grain-to grain contacts over assemblage-scale porous media domains using X-ray microtomography, Environ. Sci. Technol. 40 (12) (2006) 3762-3768.

[17] X. Li, C.-L. Lin, J.D. Miller, W.P. Johnson, Role of grain-to-grain contacts on profiles of retained colloids in porous media in the absence of an energy barrier to deposition, Environ. Sci. Technol. 40 (12) (2006) 3769-3774.

[18] W.P. Johnson, X. Li, G. Yal, Colloid retention in porous media: mechanistic confirmation of wedging and retention in zones of flow stagnation, Environ. Sci. Technol. 41 (4) (2007) 1279-1287.

[19] H. Yuan, A. Chapiro, A mathematical model for non-monotonic deposition profiles in deep bed filtration systems, Chem. Eng. J. 166 (2011) 105-115.

[20] J. Bergendahl, D. Grasso, Colloid generation during batch leaching tests: mechanics of disaggregation, Colloids Surf. A 135 (1998) 193-205.

[21] J. Bergendahl, D. Grasso, Prediction of colloid detachment in a model porous media: Hydrodynamics, Chem. Eng. Sci. 55 (9) (2000) 1523-1532.

[22] J.A. Bergendahl, D. Grasso, Mechanistic basis for particle detachment from granular media, Environ. Sci. Technol. 37 (10) (2003) 2317-2322.

[23] X. Li, P. Zhang, C.L. Lin, W.P. Johnson, Role of hydrodynamic drag on microsphere deposition and re-entrainment in porous media under unfavourable conditions, Environ. Sci. Technol. 39 (11) (2005) 4012-4020.

[24] M.M. Sharma, H. Chamoun, D.S.H. Sita Rama Sarma, R.S. Schechter, Factors controlling the hydrodynamic detachment of particles from surfaces, J. Colloid Interfaces Sci. 149 (1) (1992) 121-134.
[25] G. Ziskind, M. Fichman, C. Gutfinger, Adhesion moment model for estimating particle detachment from a surface, J. Aerosol Sci. 28 (4) (1997) 623-634.

[26] G.M. Burdick, N.S. Berman, S.P. Beaudoin, Hydrodynamic particle removal from surfaces, Thin Solid Films 488 (1-2) (2005) 116-123.

[27] M. Tong, W.P. Johnson, Excess colloid retention in porous media as a function of colloid size, fluid velocity and grain angularity, Environ. Sci. Technol. 40 (4) (2006) 7725-7731.

[28] R.P. Neyland, Thesis, Worcester Polytechnic Institute (2005).

[29] V. Canseco, A. Djehiche, H. Bertin, A. Omari, Deposition and re-entrainment of model colloids in saturated consolidated porous media: experimental study, Colloids Surf. A Physicochem. Eng. Aspects 352 (1-3) (2009) 5-11.

[30] A. Djehiche, V. Canseco, A. Omari, H. Bertin, Experimental study of colloida particles deposit in porous media: hydrodynamics and salinity effects, C.R. Mecanique 337 (9-10) (2009) 682-692.

[31] M. Elimelech, C.R. O'Melia, Kinetics of deposition of colloidal particles in porous media, Environ. Sci. Technol. 24 (10) (1990) 1528-1536.

[32] L. Gradon, Resuspension of particles from surfaces: technological, environmental and pharmaceutical aspects, Adv. Powder Technol. 20 (1) (2009) 17-28.

[33] M. Guingo, J.P. Minier, Stochastic modeling of particle deposition and reentrainment in turbulent flows, Houille Blanche 1 (2011) 82-86.

[34] J.C. Brändle de Motta, W.-P. Breugem, B. Gazanion, J.-L. Estivalezes, S. Vincent E. Climent, Numerical modelling of finite-size particle collisions in a viscous fluid, Physics of Fluids, in press.

[35] S. Vincent, J.C. Brändle de Motta, A. Sarthou, J.-L. Estivalezes, O. Simonin, E. Climent, A Lagrangian VOF tensorial penalty method for the DNS of resolved particle-laden flows, HAL (2012), 00678353.

[36] D.C. Prieve, E. Ruckenstein, Effect of London forces upon the rate of deposition of brownian particles, AIChE J. 20 (6) (1974) 1178-1187.

[37] S. Bhattacharjee, M. Elimelech, Surface element integration: a novel technique for evaluation of DLVO interaction between a particle and a flat plate, J. Colloid Interface Sci. 193 (2) (1997) 273-285.

[38] R.D. Duffadar, J.M. Davis, Interaction of micrometer-scale particles with nanotextured surfaces in shear flow, J. Colloid Interface Sci. 308 (1) (2007) 20-29.

[39] E. Martines, L. Csaderova, H. Morgan, A.S.G. Curtis, M.O. Riehle, DLVO interaction energy between a sphere and a nano-patterned plate, Colloids Surf. A Physicochem. Eng. Aspects 318 (1-3) (2008) 45-52.

[40] H. Brenner, The slow motion of a sphere through a viscous fluid towards a plane surface, Chem. Eng. Sci. 16 (3-4) (1961) 242-251.

[41] A. Ten Cate, C.H. Nieuwstad, J.J. Derksen, H.E.A. Van den Akker, Particle imaging velocimetry experiments and lattice-boltzmann simulations on a single sphere settling under gravity, Phys. Fluids 14 (11) (2002) 4012-4025.

[42] W.P. Breugem, A combined soft-sphere collision/immersed boundary method for resolved simulations of particulate flows, in: Proceedings of the ASME 2010 3rd Joint US-European Fluids Engineering Summer Meeting and 8th International Conference on Nanochannels, Microchannels, and Minichannels, FEDSM-ICNMM2010-30634. ASME, 1-5 August, Montréal, Canada 2010.

[43] J.P. Caltagirone, S. Vincent, Tensorial penalisation method for solving Navier-Stokes equations, C.R. Acad. Sci. Ser. IIb: Mecanique 329 (8) (2001) 607-613.

[44] P. Lopez, A. Omari, G. Chauveteau, Simulation of surface deposition of colloidal spheres under flow, Colloids Surf. A 240 (2004) 1-8. 